\title{
Minimally Invasive Lateral Access Corpectomy for a Solitary Renal Cell Carcinoma Spinal Metastasis: A Case Report
}

\author{
Joel Lee Zhao Jie, Tan Yu Heng Gamaliel, Wu Pang Hung
}

Department of Orthopaedic Surgery, Ng Teng Fong General Hospital, Singapore

Corresponding Author: JOEL LEE ZHAO JIE, MBBS, MRCS Department of Orthopaedic Surgery, Ng Teng Fong General Hospital, 1 Jurong East Street 21 Singapore 609606

Tel: +6591509565

E-mail: joel_lee@nuhs.edu.sg

Received: June 21, 2018

Revised: October 14, 2018

Accepted: October 29, 2018
Spinal metastases can present with neurological symptoms due to cord compression which often requires urgent surgical decompression and fixation. However, the posterior spinal approach has limitations in cord decompression. We describe our institution's experience of a Thoracic 12 corpectomy done via a minimally invasive retropleural approach for a patient with a metastatic spinal tumour. A 63 year old presented with a 1 month history of worsening atraumatic back pain with lower limb radicular pain and weakness. Magnetic Resonance Imaging (MRI) showed a T12 pathological fracture with vertebral body retropulsion causing cord compression. A Computed Tomography (CT) abdomen scan showed a heterogenous enhancing mass in the superior pole of the right kidney, suspicious of a renal cell carcinoma. He underwent initial posterior pedicle screw instrumentation from T10-L2 and a T12 bilateral laminectomy with right T12 pedicle bone biopsy, followed by a minimally invasive lateral access T12 corpectomy. His weakness improved and he had subsequent radiation therapy, chemotherapy and a right nephrectomy with good recovery. Minimally invasive lateral access retropleural approach can be considered in metastatic spinal tumour cases with cord compression as it allows for circumferential decompression while avoiding risks of anterior spinal surgery.

Key Words: Corpectomy, Lateral access, Metastatic spinal tumour

\section{INTRODUCTION}

Metastatic renal cell carcinoma (RCC) comprises $45 \%$ of all patients with renal cell carcinoma and the average survival of the patients diagnosed with metastatic RCC is four months ${ }^{1)}$. Bony metastases occur in up to $50 \%$ of patients with renal cell carcinoma ${ }^{2)}$ and are associated with a poorer prognosis ${ }^{3)}$. In particular, the prognosis is worse ${ }^{4)}$ with spinal metastases, which make up $15 \%$ of all osseous metastases ${ }^{4}$.

Spinal metastases from renal cell carcinoma may present in various ways ranging from pain to neurological symptoms such as weakness, numbness or even paralysis ${ }^{5}$. Spinal cord compression from tumour extension or pathological fracture is seen in $5-14 \%$ of patients with spinal metastases ${ }^{6)}$ and these patients often require urgent surgical decompression and fixation.

Presently, the standard surgical treatment for patients with spinal cord compression from metastases is posterior decompression via laminectomy with fixation ${ }^{7)}$. However, as majority of spinal metastases occur in the vertebral body, removal of the posterior elements fails to address the cord compression anteriorly and may lead to further spinal instability. In fact, a randomized controlled trial ${ }^{8)}$ demonstrated that patients who underwent isolated laminectomies had similar outcomes to those who were treated with radiotherapy alone. On the other hand, total en bloc vertebrectomy which allows for circumferential decompression accompanied by radiotherapy has been shown to have superior outcomes compared to radiotherapy alone ${ }^{6)}$. However the problem with the open anterior thoracic approach ${ }^{9)}$ is the association with increased morbidity due to the long duration of surgery and the presence of lung complications associated. In recent years, the minimally invasive extreme lateral approach with posterior percutaneous or open pedicle screw instrumentation has also been used successfully in the treatment of metastatic thoracic and lumbar vertebral body tumours ${ }^{10)}$.

We describe our institution's experience of a case of T12 corpectomy done via a minimally invasive direct lateral retropleural approach in a patient with metastatic renal cell carcinoma with a T12 pathological fracture causing spinal cord compression.

\section{CASE REPORT}

A 63-year-old Chinese male presented with a 1-month history of worsening atraumatic lower back pain with bilateral lower 
limb posterior radicular pain and subjective weakness. He had a known history of an atraumatic T12 compression fracture diagnosed on a spinal radiograph 3 weeks prior to presentation. On admission, he was noted to have mild left hip flexion weakness (MRC (Medical Research Council) grade 4) and left knee extension weakness (MRC grade 4). Otherwise, lower limb sensation was preserved bilaterally in dermatomes L2-S1 and bladder/bowel function was normal. Magnetic Resonance Imaging (MRI) of the whole spine revealed a T12 pathological fracture with vertebral posterior cortex retropulsion and heterogeneous extradural soft tissue causing cord compression (Fig. 1) ${ }^{1)}$

He then underwent staged resection of his T12 tumor with stage one consisting of posterior minimally invasive pedicle screw instrumentation from $\mathrm{T} 10$ to $\mathrm{L} 2$ under image intensifier guidance and a T12 bilateral laminectomy with bone biopsy of the right T12 pedicle. It was noted that the initial bone biopsy was negative. This was attributed to a sampling error as the bone biopsy was performed via a core biopsy needle through the pedicle during the posterior approach. The decision for posterior pedicle screw instrumentation was made to provide posterior support and stabilization in the setting of a pathological fracture where fusion could be unreliable. The minimally invasive technique was employed to reduce excessive soft tissue dissection. A computed tomography (CT) scan of the thorax, abdomen and pelvis showed a heterogeneous enhancing mass in the superior pole of the right kidney (measuring $4.1 \times 4.2 \times 3.2 \mathrm{~cm}$ ) that was suspicious for a renal cell carcinoma (Fig. 2).

While awaiting the second staged decompression surgery, he developed worsening bilateral lower limb weakness and radicular pain on post-operative day 12 and examination revealed new weakness over left ankle dorsiflexion (MRC grade 2), left toe dorsiflexion (MRC grade 2), left foot plantarflexion (MRC grade 2). A repeat MRI scan of the whole spine showed severe cord compression at the T12-L1 level with edema secondary to the retropulsed posterior cortex of the T12 vertebral body as well as severe narrowing of the left T11-12 neural foramen (Fig. 3). The patient then underwent a T12 corpectomy via a minimally invasive lateral retropleural approach with anterior decompression and the placement of an expandable cage. The technique is described below.

\section{Operative Technique of Minimally Invasive Lateral Access Corpectomy}

Preoperative embolization of feeding vessels to the tumour was performed. Patient underwent general anaesthesia with intravenous propofol and was positioned in the right lateral position with tape securing the pelvis and the chest. Neuro-monitoring was in place. Pre-operative Image Intensifier imaging was done to mark out the level of resection. A $5 \mathrm{~cm}$ Incision was made along the rib overlying the corpus of T12 and the overlying rib harvested as bone graft. This was done by first using the Alexander periosteal elevator followed by the guillotine rib cutter, carefully protecting the neurovascular structures in the inferior aspect of the rib. Careful gentle dissection should be made to preserve the visceral pleura and parietal pleura.
A retropleural approach was done in the incidence using gentle finger and Kittner blunt dissection to reach the posterior aspect of T12 rib head. The lung with its intact pleura was gently separated and retracted anteriorly using the lung retractor as part of the Maxcess-4 retractor system and the Nuvasive XLIF corpectomy system. The superior and inferior disc was identified. The MIS posterior blade of the retractor is placed docking over the rib head, with the lung retractor over the anterior border of T12 corpus, superior blade over the inferior end plate of T11 and inferior blade over the superior end plate of L1 (Fig. 4A). Full discectomy was performed using cobb elevator and pituitary rongeur (Fig. 4B), taking care to complete the dissection over the contralateral annulus first over the T11/T12 disc followed
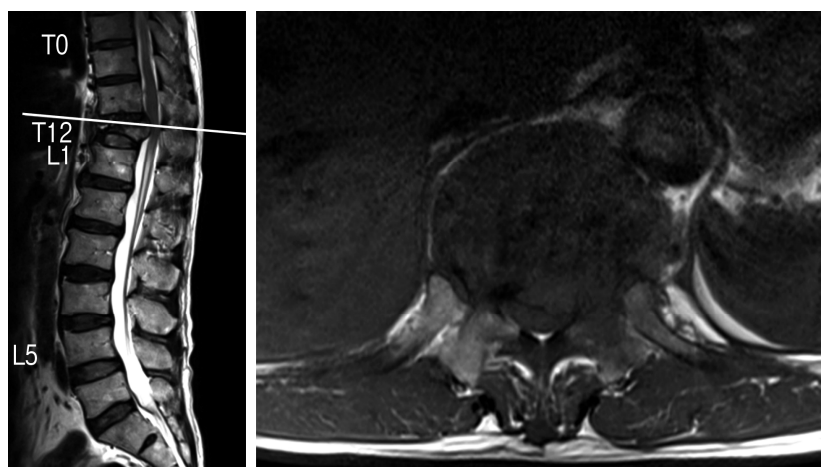

Fig. 1. T2 weighted Sagittal and Axial cuts at T12 (MRI whole spine).

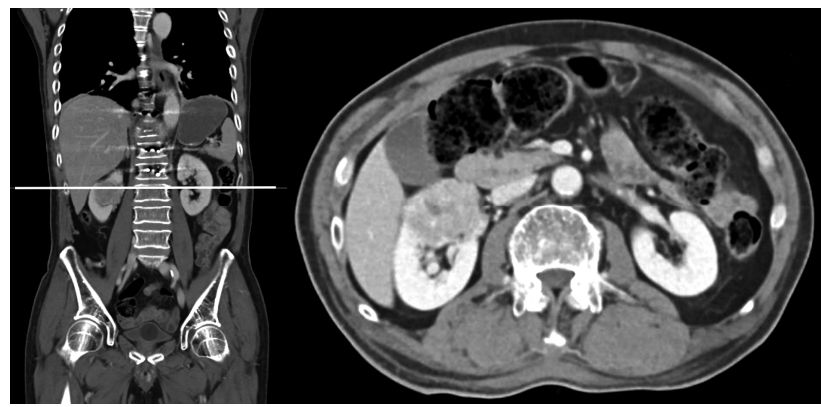

Fig. 2. Coronal and Axial cuts (CT Thorax, Abdomen and Pelvis).

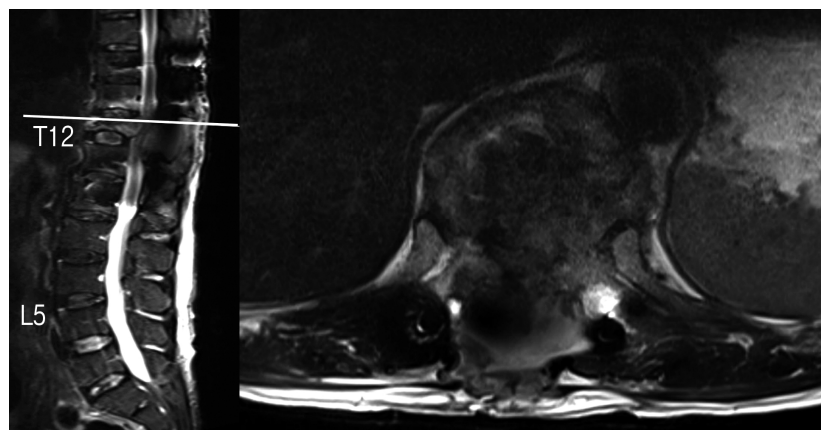

Fig. 3. T2 weighted Sagittal and Axial cuts at T12 (MRI whole spine). 
by T12/L1 disc. The rib head was transected using sharp osteotome. The left pedicle was completely transected using a sharp osteotome with the last sleeve of bone removed using Kerrison 2 Rongeur, taking care to protect the neural element without too much traction or any blunt injury to the cord. The contralateral pedicle was transected to half of the contralateral pedicle. The anterior longitudinal ligament was separated from the anterior corpus using Penfield dissector and Macdonald dissector. As the tumor was soft and T12 had fractured, piecemeal removal of T12 using a rongeur was done with care to protect neural element posterior and vessels anteriorly. This was sent for histology testing. After ensuring complete removal of corpus of the T12 vertebra, sizing was done using Nuvasive expandable cage trial and a Nuvasive XCORE II CORE 21-27 mm and Endplate $18 \times 40 \mathrm{~mm}$ (parallel endplate) expandable cage was inserted (Fig. 4C, 4D, 4E \& 4F) Osteocel Pro 5 cc bone graft was used. Fusion was considered in view that this was a solitary metastasis from renal cell carcinoma with possible good survival.
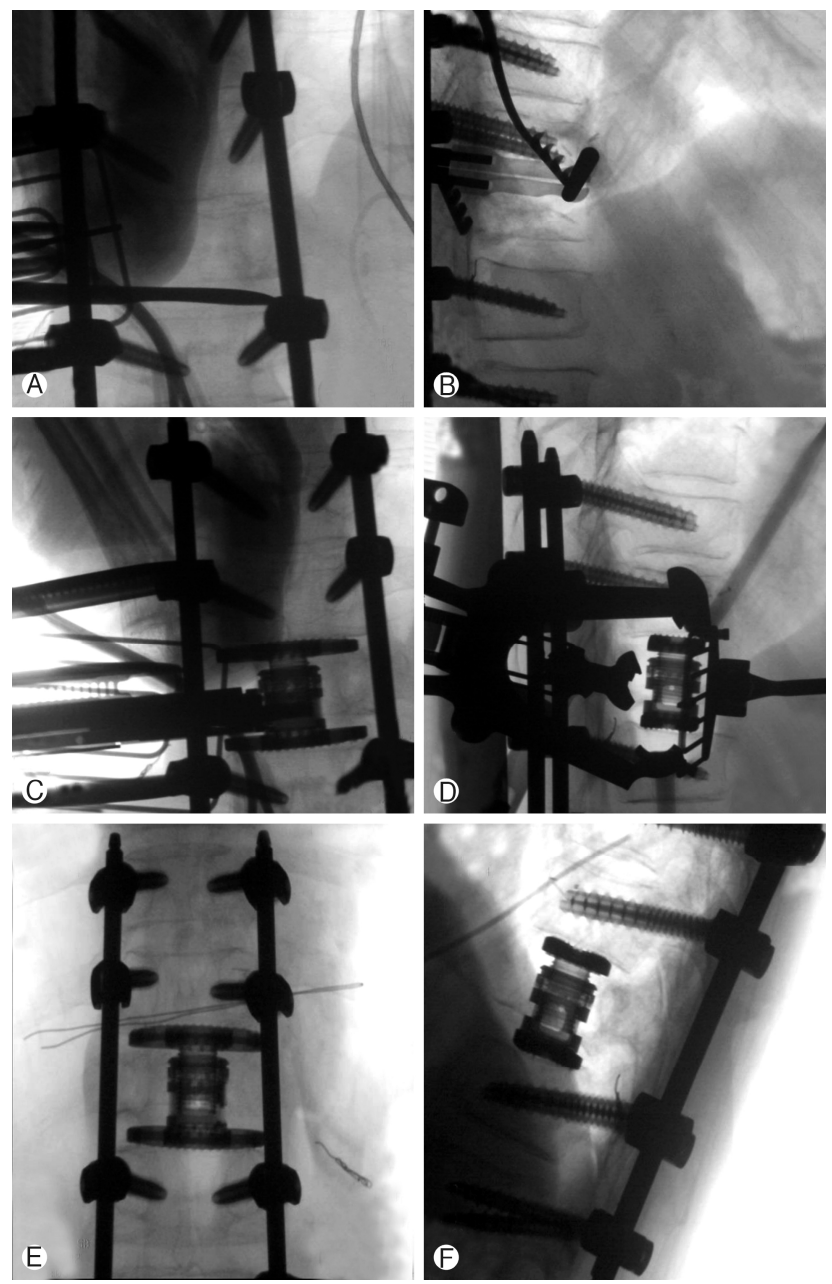

Fig. 4. (A) Docking Image. (B) Discectomy at T12/L1 using Cobb elevator. (C) Insertion of expandable cage (AP). (D) Insertion of expandable cage (lateral). (E) Post- expandable cage insertion $(A P)$. (F) Post- expandable cage insertion (lateral).
The author accepts that it is a controversial decision in view of follow up radiation that may make fusion difficult. The wound was closed in layers. A chest drain inserted with a separate stab incision over the T10/11 Intercostal space anterior to mid clavicular line under direct visual guidance. Wound was closed in layers. The patient was then turned prone for evacuation of the posterior dense hematoma and for further lateral and posterior decompression at the T12 level.

Post operatively, patient did well and mobilized next morning, Chest drain stopped bubbling on post-operative day 2 and is removed. He was discharged 9 days later and his wound healed on post-operative day 14 . He was reviewed by radiation oncology and was commenced on intensity-modulated radiotherapy (IMRT) on post-operative day 31 (50 Gy), day 33 and day 36 . He was continued on radiation therapy for a total of 5 weeks. He then underwent right laparoscopic radical nephrectomy approximately 5 months post-spinal surgery with good recovery postoperation. He was last reviewed in the spine clinic 10 months
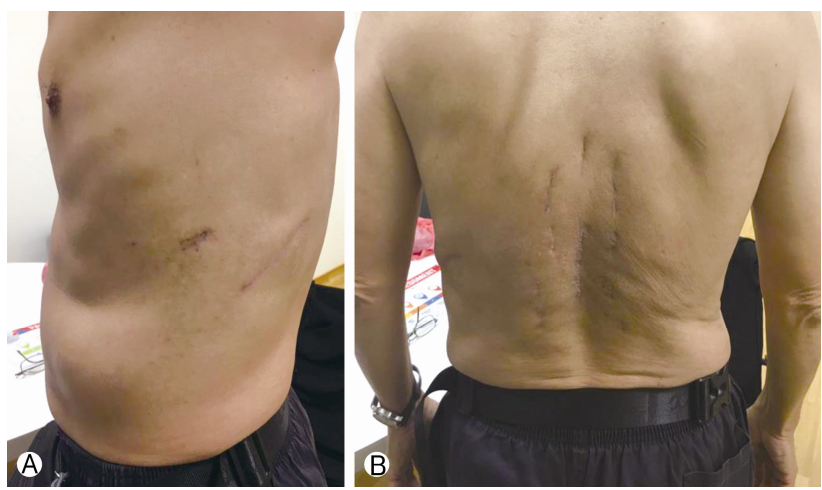

Fig. 5. (A) Lateral Access corpectomy scar. (B) Posterior spinal surgical scars.
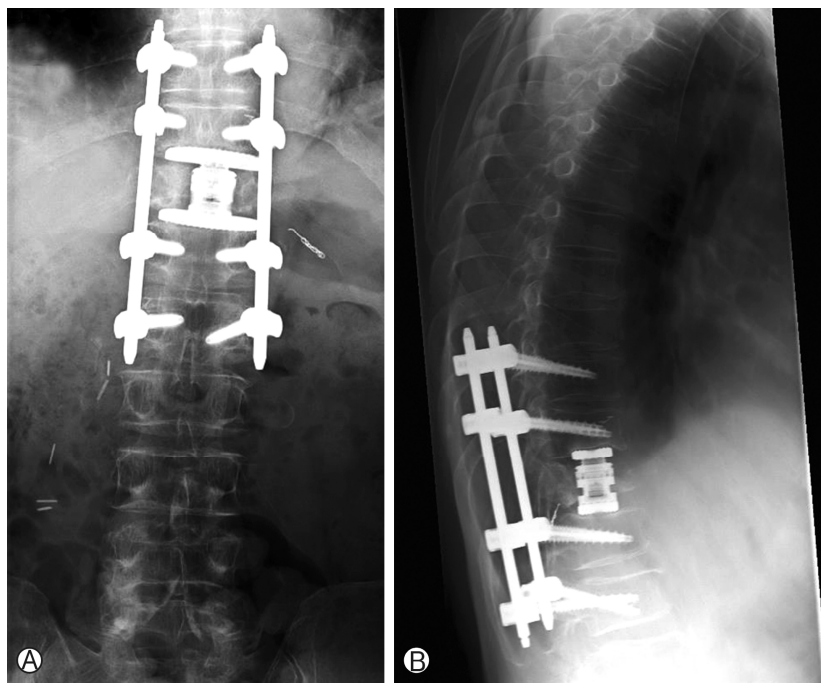

Fig. 6. (A) AP and (B) lateral post-operative spinal radiographs (5 months post-op). 
post-spinal surgery with no back pain and no lower limb radicular pain, weakness or numbness. Lower limb neurological examination of power and sensation was normal (MRC grade 5) and there were no wound complications (Fig. 5). The latest spinal radiographs also did not reveal any hardware complications (Fig. 6). The patient has returned to work as a mechanic and is on follow-up with urology, radiation oncology and medical oncology.

\section{Discussion}

Management of spinal metastatic tumours is individualized to the patient depending on the prognosis and the presence of spinal cord compression. Treatment can be nonsurgical with chemotherapy, radiotherapy or immunotherapy or surgical treatment, which has been the only recognized therapy to improve quality of life in patients with spinal metastases from renal cell carcinoma ${ }^{5}$. Moreover, in patients with solitary vertebral metastasis from renal cell carcinoma, en bloc resection has been shown to improve their overall survival time ${ }^{1)}$.

En bloc resection can vary from sagittal resection, posterior resection or vertebrectomy $y^{5}$. In recent years, vertebrectomy with anterior column reconstruction has been gaining in popularity ${ }^{5)}$ given its ability to provide circumferential spinal cord decompression. This can be done either with a combined posterior and anterior approach or a posterior-only approach ${ }^{5}$. Although a posterior-only surgical approach can potentially reduce surgical time and blood loss, this technique has a high risk of nerve root and spinal cord damage and the difficulty in reconstructing the anterior column ${ }^{5}$. Presently, a combined anterior transthoracic approach to the anterior spinal column with subsequent posterior instrumentation for en bloc resection for thoracic spinal metastases has been used with good results ${ }^{6}$. This is commonly done in the same setting as a staged procedure for spinal circumferential spinal fusion has been shown to have no mortality benefit and may instead increase risk of complications such as adult respiratory distress syndrome (ARDS) and deep venous thrombosis (DVT) ${ }^{11)}$.

The anterior transthoracic spinal approach, though allowing for the placement of a sizeable anterior spinal graft/implant with potential benefits in reduced subsidence ${ }^{9}$, has been associated with morbidity due to the need for thoracotomy ${ }^{12)}$. Approach-related complications include pulmonary contusion, atelectasis, pleural effusion, hemothorax and are considerable ranging from 14.1 to $29.4 \%$ even with the use of thoracoscopy ${ }^{12}$. This approach is also limited by the late visualization of the neural elements ${ }^{13)}$ which may be inadvertently injured during the dissection process.

The minimally invasive lateral retropleural approach is one that accesses the anterior spine through a small lateral chest incision and utilizes a unique retraction system so as not to disrupt the posterior vertebral elements or spinal musculature ${ }^{10)}$. This approach has been used successfully for vertebrectomy in the setting of traumatic thoracolumbar fractures ${ }^{14)}$. In recent years, this approach has also been used effectively in the treatment of spinal metastatic tumours ${ }^{10)}$. This approach has the advantages of reduced blood loss, shortened hospitalization and reduced risk of wound infection or dehiscence ${ }^{10)}$. As this approach is retropleural, there is also a significantly reduced risk of diaphragmatic and pleural injury ${ }^{12)}$ with a cadaveric study on 9 individuals demonstrating that the diaphragmatic fibers are not cut during tubular dilation during the exposure phase ${ }^{15)}$. However, it must be said that this approach may not be suitable for all patients and patient selection with the appropriate anatomy is key.

Before or after spinal surgery, many patients with metastatic spinal tumours are required to go for radiotherapy to reduce the risk of local recurrence. A systemic review by Itshayek et al. ${ }^{16)}$ showed that post-operative radiotherapy was not a statistically significant risk factor in causing surgical complications and only 8 out of 51 case series involving patients with spinal metastases receiving post-surgical radiotherapy reported woundrelated complications, with an average of 3.9\% (range 0-16\%) ${ }^{16)}$. However, the review recommended avoiding radiotherapy for a week or longer after surgical treatment for spinal metastases on the grounds of general principles of wound biology as well as a study by Ormsby et al. ${ }^{17)}$ on patients with soft tissue sarcomas which demonstrated a delay in after-loading of catheters with radioactive sources to at least the fifth postoperative day has decreased the significant wound healing complications associated with brachytherapy.

\section{CONCLUSION}

A minimally invasive retropleural lateral approach for thoracic corpectomy can be used safely and successfully in patients with metastatic spinal tumours with cord compression.

\section{REFERENCES}

1. Thyavihally BY, Mahantshetty U, Chamarajanagar RS, Raibhattanavar SG, Tongaonkar HB: Management of renal cell carcinoma with solitary metastasis. World J Surg Onc 3(48), 2005

2. Maldazys JD, De Kernion JB: Prognostic factors in metastatic renal carcinoma. J Urol 136:376-379, 1986

3. Beuselinck B, Oudard S, Rixe O, Wolter P, Blesius A, Ayllon J, et al: Negative impact of bone metastasis on outcome in clearcell renal cell carcinoma treated with sunitinib. Ann Oncol 22: 794-800, 2011

4. Althausen P, Althausen A, Candace Jennings L, Mankin HJ: Prognostic factors and surgical treatment of osseous metastases secondary to renal cell carcinoma. Cancer 80:1103-1109, 2011

5. Gasbarrini A, Esteves SC, Cappuccio M and Boriani SL: Management of renal cell carcinoma metastasis of the spine, emerging research and treatments in renal cell carcinoma 2012. Dr Amato R (Ed.)

6. Patchell RA, Tibbs PA, Regine WF, Payne R, Saris S, Kryscio $\mathrm{RJ}$, et al: Direct decompressive surgical resection in the treatment of spinal cord compression caused by metastatic cancer: a randomized trial. Lancet 366:643-648, 2005

7. Langdon J, Way A, Heaton S, Bernard S, Molloy S: The management of spinal metastases from renal cell carcinoma. Ann R Coll Surg Engl 91:649-652, 2009 
8. Young RF, Post EM, King GA: Treatment of spinal epidural metastases. Randomized prospective comparison of laminectomy and radiotherapy. J Neurosurg 53:741-748, 1980

9. Jandial R, Chen YM: Modified lateral extracavitary approach for vertebral column resection and expandable cage reconstruction of thoracic spinal metastases. Eur Spine J 24(Suppl 3): 353-360, 2015

10. Serak J, Vanni S, Levi AD: The extreme lateral approach for treatment of thoracic and lumbar vertebral body metastases. J Neurosurg Sci 3, 2015

11. Passias PG, Yan M, Chiu YL, Mazumdar M, Girardi FP, Memtsoudis SG: Comparative Safety of Simultaneous and Staged Anterior and Posterior Spinal Surgery. Spine 37(3):247-255, 2012

12. Lall RR, Smith ZA, Wong AP, Miller D, Fessler RG: Minimally invasive thoracic corpectomy: surgical strategies for malignancy, trauma, and complex spinal pathologies. Minim Invasive Surg 213791, 2012

13. Sasso RC, Kenneth Burkus J, LeHuec JC: Retrograde ejacula- tion after anterior lumbar interbody fusion: transperitoneal versus retroperitoneal exposure. Spine (Phila Pa 1976) 28:10231026, 2003

14. Smith WD, Dakwar E, Le TV, Christian G, Serrano S, Uribe J: Minimally invasive surgery for traumatic spinal pathologies: a mini-open, lateral approach in the thoracic and lumbar spine. Spine (Phila Pa 1976) 35(26 Suppl):S338-346m, 2010

15. Dakwar E, Ahmadian A, Uribe JS: The anatomical relationship of the diaphragm to the thoracolumbar junction during the minimally invasive lateral extracoelomic (retropleural/retroperitoneal) approach. Journal of Neurosurgery 16(4):359-364, 2012

16. Itshayek E, Yamada J, Bilsky M, Schmidt M, Shaffrey C, Gerszten $\mathrm{P}$, et al: Timing of surgery and radiotherapy in the management of metastatic spine disease: A systematic review. International Journal of Oncology 36:533-544, 2010

17. Ormsby MV, Hilaris BS, Nori D: Wound complications of adjuvant radiation therapy in patients with soft-tissue sarcomas. Ann Surg 210:93-99, 1989 